\title{
The translation and validation of the Arabic Version of the Polycystic Ovary Syndrome Health-Related Quality of Life Questionnaire (AR-PCOSQ)
}

\author{
Sultan Alghadeer ${ }^{1,2^{*}}$ (D) Alhanouf Algarawi ${ }^{2}$, Faten Abu-Rkybah², Mashael M. Alshebly ${ }^{3,4}$ and Yazed Alruthia ${ }^{2}$
}

\begin{abstract}
Background: Polycystic ovarian syndrome (PCOS) is a hormonal disorder that is prevalent in females of reproductive age with signs and symptoms that significantly reduce self-esteem and have a negative impact on their quality of life. The management of PCOS signs and symptoms should result in an improvement in the health-related quality of life (HRQoL) of patients. Polycystic ovarian syndrome questionnaire (PCOSQ) is a disease-specific scale. The PCOSQ has been translated into different languages and assessed in different populations. The validity and reliability of PCOSQ varied depending on the ethnicity and culture of the respondents. The objective of the study was to establish a valid and reliable version of the PCOSQ (AR-PCOSQ) in Arabic.
\end{abstract}

Methods: A cross-sectional study using the translated and validated AR-PCOSQ questionnaire was conducted by interviewing 117 women with PCOS.

Results: The mean age (years) and BMI $\left(\mathrm{kg} / \mathrm{m}^{2}\right)$ of subjects were $29.90 \pm 6.33$ and $27.21 \pm 5.54$, respectively. Most of the patients had $\geq 1$-year long history of PCOS (73.5\%) and a post-school degree (64.96\%). The content validity index (CVI) for the AR-PCOSQ from 10 gynecologists was 0.9 , indicating satisfactory validity content. The internal consistency for reliability confirmation measured by Cronbach's alpha coefficient was applied. Alpha coefficients for all items together was 0.863 , indicating good reliability. The intraclass correlation coefficients for each item for 30 participants were also acceptable, ranging from 0.911 to 0.986 with $p$ value $<0.001$. As far as the factor analysis is concerned, the overall Kaiser-Meyer-Olkin sampling adequacy measure was 0.772 . The Bartlett sphericity test was significant $(p \leq 0.001)$, Indicating that there were interrelated variables.

Conclusion: Our results demonstrated the initial reliability and validity of the Arabic version of the PCOSQ as a measure of specific HRQOL in Saudi women with PCOS. This will fill an important gap in measuring the HRQoL for patients with PCOS in research and community settings in Saudi Arabia. The AR-PCOSQ can be used to help prioritize healthrelated concerns from the patient's perspective.

Keywords: Polycystic ovarian syndrome, Arabic, Questionnaire, Translation, Validity, Reliability

*Correspondence: salghadeer@ksu.edu.sa

${ }^{1}$ Basic Sciences Department, Prince Sultan College for Emergency

Medical Services, King Saud University, Riyadh, Saudi Arabia

Full list of author information is available at the end of the article

\section{Background}

One of the prevalent endocrine disorders among women of reproductive age is polycystic ovarian syndrome (PCOS). The global prevalence of PCOS is estimated to range between 5 and $10 \%$; however, its prevalence in Saudi Arabia is largely unknown [1, 2]. This syndrome 
includes a myriad of signs and symptoms such as amenorrhea or oligomenorrhea, obesity, infertility, anovulation, acne, and hirsutism [3, 4]. These signs and symptoms can significantly lower patients' self-esteem and negatively impact their physical quality of life $[4,5]$. Furthermore, PCOS negatively affects the mental wellbeing of the affected patients resulting in both depression and anxiety [6]. Therefore, managing PCOS signs and symptoms should result in an improvement in patients' health-related quality of life (HRQoL) [7].

Measuring HRQoL for PCOS patients at baseline as well as after the impact of different treatment approaches is informative and helpful to clinicians and patients alike to determine the value of each treatment approach from the patients' perspective [8].

Today, many clinicians, health care policy makers, and regulators are interested in Patient-Reported Outcomes (PROs) to assess the quality of provided care. Of those PROs, HRQoL is considered one of most important measures in assessing the quality of care. Therefore, to evaluate the HRQoL among different patient populations, several scales have been developed These scales can be generic (whereby they are used to assess the HRQoL among patients with different medical conditions) or disease-specific [9]. Generic HRQoL scales such as the Short Form Health Survey (SF-36) or abbreviated version of World Health Organization Quality of Life (WHOQOL-BREF) scale may not be as accurate as the disease-specific HRQoL scales in measuring the HRQoL among patients with specific health care conditions such as PCOS [10]. PCOS-specific HRQoL scales such as the polycystic ovarian syndrome questionnaire (PCOSQ) have been developed to address different domains in patient HRQoL relevant to PCOS, since there is no specific HRQoL instrument to use in women with PCOS.

Despite its specificity, different HRQoL outcomes among women with PCOS from different backgrounds confirm that in women with PCOS, ethnicity and culture play an important role in evaluating the quality of life. Chinese, Korean, South African, and Swedish versions of the PCOSQ were found to be reliable, valid, and culturally acceptable [11-14]. In the UK, PCOSQ was found to be reliable, but its validity needs to be improved by incorporating a dimension on acne [15]. In Iran, except for menstrual issues, the questionnaire was found to be accurate and valid in all aspects $[16,17]$.

The PCOSQ could be used to identify issues associated with the HRQoL in women with PCOS, evaluate the full effectiveness of treatment regimes, identify and report changes in patients' health status over time, and generate more understanding of the impact that the symptoms and treatments of PCOS could have against HRQoL. However, a variation in validity and reliability of PCOSQ was noticed among women from different ethnicities and cultures. The aim of this study was to establish a valid and reliable Arabic version of the PCOSQ.

\section{Methods \\ Study design and participants}

A cross-sectional study using the translated questionnaire (AR-PCOSQ) was conducted by interviewing 117 PCOS women who attended King Khalid University Hospital (KKUH) obstetrics and gynecology clinics during the period from November to December 2017. The institutional review board (IRB) at KKUH granted ethical approval (IRB Project No. E-17-2545). The criteria for inclusion were 18-45 years of age, married, Arabic-speaking women with no verbal communication difficulties. Non-classic adrenal hyperplasia, thyroid dysfunction, hyperprolactinemia, previously diagnosed diabetes, any drug with an effect on insulin levels, or hormonal drugs (including contraceptive pills) were excluded at least two months before participating.

\section{Measurement}

A specific tool to evaluate the HRQoL of women with PCOS is PCOSQ. It is composed of 26 items categorized into five sections: emotions (eight items), body hair (five items), weight concerns (five items), infertility concerns (four items), and menstrual irregularities (four items). Each item is scored with a seven-point Likert rating system where seven represents the best situation and 1 represents the worst situation. The emotions section asks questions related to mental illness, depression, mood, self-esteem, and fear of cancer as consequence of PCOS diagnosis. The body hair section contains items concerning noticeable hair in face and overabundance of hair throughout the body. The issues of gaining weight or being overweight and infertility issues such as inability to conceive and having children are included in the weight and infertility concerns sections, respectively. The menstrual irregularities section includes items regarding abnormalities in menstrual periods and body discomforts secondary to menstrual abnormalities. The mean score for all items in a section indicates the score for each woman [18].

\section{Procedure}

Two interviews were conducted to assess the face validity and test-retest reliability. The first interview was face-toface interview while the second interview was conducted subsequently via the phone during an interval period of 5 days to 2 weeks post the face-to-face interview. This interval was selected in order to maintain the consistency and similarity of PCOS condition as the quality of 
life changes when PCOS condition changes with time progress.

Use of the Polycystic Ovary Syndrome Questionnaire, authored by Dr. Gordon Guyatt, et al. was made under license from University, Hamilton, Canada, with the permission of the copyright owner, The Endocrine Society, Maryland, USA. A forward-backward process has been used to translate the English version of the PCOSQ into Arabic. Two bilingual translators (one with a medical experience and one without) proficiently fluent in English each independently translated the complete English version of the PCOSQ into Arabic, including item content, response options, and instructions. The two forward translations were merged into one version by primary investigators after the expert panel discussions that consist of gynecologists, professional translators, and experts in public health. Any discrepancies between the forward translations will be identified and resolved by the expert panel. Two translators independently later translated the single forward translation back into English in the backward translation while totally blinded to the PCOSQ's original English version.

\section{Statistical analysis}

Using IBM SPSS Statistics, statistical analysis was carried out. To test the validity of the Arabic translated version of PCOSQ, face and content validity was used. Reliability analysis of reliability of the test-retest as well as internal consistency were carried out.

\section{Content validity}

The content validity index (CVI) of the items was calculated based on individual consideration or feedback on our scale relevancy by a group of 10 gynecologists. The relevancy of the items was assessed using a four-point Likert scale: (1) not relevant, (2) somewhat relevant, (3) relevant, (4) very relevant. The recommended acceptable lower limit for CVI is 0.80 [19].

\section{Face validity}

To test validity, to ensure the linguistic and conceptual equivalence of the translation, and to confirm the accuracy, appropriateness, and interpretation of the translated questionnaire, AR-PCOSQ was given to 30 patients with PCOS [20].

\section{Internal consistency}

Internal consistency relates to a tool homogeneity. Cronbach's alpha coefficient value from 0 to 1 was utilized to evaluate the internal consistency. High value means that used tool is more reliable with low predictable errors. Cronbach's alpha coefficient value of $\geq 0.7$ indicates acceptable internal consistency for the used tool [21].

\section{Test-retest reliability}

Intraclass correlation-coefficient (ICC) value from 0 to 1 was utilized to evaluate the test-retest reliability that aims to determine the consistency of used tool when the same tool it is applied to the same participants at two different times. The following categories were selected to interpret the agreement levels: $00-0.2$ as small, $0.21-0.40$ as fair, $0.41-0.60$ as moderate, $0.61-0.80$ as substantial and $0.81-1$ as almost perfect [22].

\section{Factor analysis}

Factor analysis is a statistical tool to explain the ARPCOSQ subscale factor structure variability. It is used to simplify the interpretation of factors and minimize the number of variables affected by each factor. The factor structure of the AR-PCOSQ was extracted by utilizing exploratory factor analysis (EFA) [23]. Kaiser-MayerOlkin (KMO) measure of adequacy in sampling and Bartlett's test were used as well. KMO value greater than 0.6 indicates the sample is adequate $[24,25]$.

\section{Results}

A total of 117 women with PCOS have been enrolled in the study. The mean age (years) and BMI $\left(\mathrm{kg} / \mathrm{m}^{2}\right)$ of subjects were $29.9 \pm 6.33$ and $27.21 \pm 5.54$, respectively. Most of the patients had $\geq 1$ year long history of PCOS (73.5\%) and a post-school degree (64.96\%) (Table 1).

\section{Content validity}

Ten gynecologists (4 males; and 6 females) whose ages range from 40 to 62 with at least 10 years of experience were contacted. The content validity index (CVI) for the AR-PCOSQ from 10 gynecologists was 0.9 , indicating satisfactory validity content. The ten gynecologists rated almost all the translated items as relevant clinically and culturally with very few comments on some items that were slightly modified.

\section{Table 1 Socio-demographic and clinical characteristic} $(n=117)$

\begin{tabular}{ll}
\hline Characteristics & \\
\hline Age $($ years), mean \pm SD & $29.90 \pm 6.33$ \\
BMI $\left(\mathrm{kg} / \mathrm{m}^{2}\right)$, mean \pm SD & $27.21 \pm 5.54$ \\
History of PCOS (years), $n(\%)$ & \\
$<1$ year & $31(26.5 \%)$ \\
$\geq 1$ year & $86(73.5 \%)$ \\
Education level, $n(\%)$ & \\
Pre-secondary school & $3(2.56 \%)$ \\
Secondary school & $38(32.48 \%)$ \\
Post-secondary school & $76(64.96 \%)$ \\
\hline
\end{tabular}

$S D$ standard deviation 


\section{Face validity}

Thirty patients were interviewed for content validity. Their mean ages (years) and BMI $\left(\mathrm{kg} / \mathrm{m}^{2}\right)$ were $29 \pm 7.08$ and $26.7 \pm 5.51$, respectively. Most of the thirty patients had $\geq 1$ year long history of PCOS (21 patients; $70 \%)$ and a post-school degree (17 patients; 56.7\%). After the interview, it was ensured that the ARPCOSQ was appropriate and relevant clinically and culturally, and that it was simple and understandable linguistically with no changes in wording required.

\section{Reliability}

The internal consistency for reliability confirmation measured by Cronbach's alpha coefficient was applied to all participants $(n=117)$. For each subscale and over all items, Cronbach's alpha Reliability coefficient was calculated. As shown in Table 2, all alpha coefficients (except that for menstrual problems) exceeded the recommended value of 0.70 . Also, the alpha coefficients for all items together was 0.863 , indicating good reliability. The intraclass correlation coefficients for each item for the 30 participants were also acceptable, ranging from 0.911 to 0.986 with $p$ value $<0.001$ (Table 3 ).

\section{Factor analysis}

The AR-PCOSQ was studied by conducting the key component analysis. The overall Kaiser-Meyer-Olkin sampling adequacy test was 0.772 . The sphericity test of the Bartlett was significant $(p \leq 0.001)$ indicating that the variables correlated with one another. The ARPCOSQ was found to have six factors. The number of explained variances ranged from 4.57 to $24.22 \%$, with $64.44 \%$ of the overall variance explained (Table 4). Table 5 shows the factor loading from the AR-PCOSQ principal component analysis. The loading factor of the items is all greater than 0.514 . In the last six items, different items were loaded in different factors.

Table 2 Cronbach's alpha of the AR-PCOSQ subscales and over all items $(n=117)$

\begin{tabular}{llll}
\hline Subscales & Cronbach's alpha & Mean & SD \\
\hline Emotion and feelings & 0.723 & 3.70 & 1.28 \\
Body hair & 0.888 & 4.01 & 2.08 \\
Weight & 0.849 & 3.65 & 1.88 \\
Infertility & 0.719 & 4.05 & 1.72 \\
Menstrual problem & 0.697 & 3.02 & 1.40 \\
Over all items & 0.863 & 3.67 & 1.08 \\
\hline
\end{tabular}

SD standard deviation

\begin{tabular}{lccc}
$\begin{array}{l}\text { Table } 3 \\
\text { of the AR-PCOSQ subscales }(\mathbf{n}=\mathbf{3 0})\end{array}$ & (ICC) \\
\hline Subscales & ICC & $\begin{array}{l}\text { Mean test } \\
\text { Mean re-test }\end{array}$ & $\begin{array}{l}\text { SD test } \\
\text { SD re-test }\end{array}$ \\
\hline Emotion and feelings & $0.986^{*}$ & 23.67 & 10.08 \\
& & 23.17 & 9.11 \\
Body hair & $0.986^{*}$ & 18.87 & 10.31 \\
& & 18.37 & 9.43 \\
Weight & $0.951^{*}$ & 16.87 & 9.50 \\
& & 16.60 & 8.06 \\
Infertility & $0.979^{*}$ & 16.90 & 7.56 \\
& & 16.67 & 7.44 \\
Menstrual problem & $0.911^{*}$ & 12.70 & 7.79 \\
& & 10.83 & 6.86
\end{tabular}

$S D$ standard deviation

${ }^{*} p$ value $<0.001$

\section{Discussion}

According to our findings of the PCOSQ in Arabicspeaking women, the Arabian PCOSQ version was culturally acceptable, applicable, and seemed relevant to their conditions. It has shown that the AR-PCOSQ version was overall relevant to all PCOS-related concerns as it expressed by our subjects. Despite of the fact that no item was recognized as missing, some items may be introduced or amended with further studies. In previous studies, acne domain was introduced as an essential domain to be involved into the PCOSQ as important factor to consider for assessing the wellbeing of women with PCOS $[8,17]$.

The satisfied internal consistency of our translated questionnaire (AR-PCOSQ) was obtained in almost all domains (body hair, emotions, weight, and infertility problems) as each domain scored above 0.7 on Cronbach's alpha correlation coefficient. However, the menstrual domain of the AR-PCOSQ scored 0.69 which is considered acceptable internal correlation coefficient according to George and Marley [26]. Similar studies among women from the United Kingdom, Canada, and

\section{Table 4 Total variance explained}

\begin{tabular}{lc}
\hline Factor & \% of variance \\
\hline 1 & 24.22 \\
2 & 12.92 \\
3 & 8.79 \\
4 & 8.26 \\
5 & 5.68 \\
6 & 4.57 \\
Total variance explained & 64.44 \\
\hline
\end{tabular}


Table 5 Factor loadings from the AR-PCOSQ principal component analysis

\begin{tabular}{|c|c|c|c|c|c|c|c|}
\hline \multirow{2}{*}{$\begin{array}{l}\text { Q\# and belonged subscale } \\
\text { in the original questionnaire }\end{array}$} & \multirow[t]{2}{*}{ Item } & \multicolumn{6}{|l|}{ Factor } \\
\hline & & 1 & 2 & 3 & 4 & 5 & 6 \\
\hline Q16:H & Embarrassment about excessive body hair & 0.889 & 0.039 & -0.036 & 0.174 & 0.089 & -0.024 \\
\hline Q26: H & Growth of visible hair & 0.866 & 0.030 & -0.077 & 0.184 & 0.158 & 0.013 \\
\hline Q15:H & Growth of visible hair on face & 0.857 & 0.088 & -0.024 & 0.033 & 0.065 & 0.019 \\
\hline Q9: H & Growth of visible hair on upper lip & 0.748 & 0.056 & -0.004 & 0.060 & 0.120 & 0.222 \\
\hline Q1:H & Growth of visible hair on chin & 0.691 & 0.110 & 0.051 & -0.048 & -0.136 & 0.399 \\
\hline Q10:W & Trouble dealing with weight & 0.171 & 0.799 & 0.010 & 0.068 & -0.053 & 0.117 \\
\hline Q3:W & Concern of being overweight & -0.046 & 0.791 & -0.045 & 0.070 & 0.158 & 0.122 \\
\hline Q12:W & Felt frustration in trying to lose weight & -0.009 & 0.788 & 0.073 & 0.245 & 0.020 & 0.030 \\
\hline Q24:W & Have difficulty staying at your ideal weight & 0.167 & 0.770 & 0.165 & 0.000 & 0.100 & 0.133 \\
\hline Q22:W & Feel like not sexy as result of overweight & 0.029 & 0.641 & 0.064 & 0.461 & 0.110 & -0.057 \\
\hline Q13:। & Felt afraid of not being able to have children & 0.000 & 0.050 & 0.907 & 0.076 & 0.012 & 0.021 \\
\hline Q5: I & Concerned with infertility problems & -0.092 & 0.009 & 0.903 & 0.077 & 0.012 & 0.034 \\
\hline Q25: I & Feel sad because of infertility problems & -0.010 & 0.113 & 0.824 & 0.054 & 0.011 & -0.109 \\
\hline Q6: E & Moody as result of PCOS & 0.124 & 0.183 & 0.046 & 0.736 & 0.127 & 0.079 \\
\hline Q2: E & Depressed for having PCOS & 0.121 & 0.078 & 0.108 & 0.704 & 0.195 & 0.278 \\
\hline Q11:E & Low self-esteem as result of PCOS & -0.006 & 0.189 & 0.193 & 0.586 & -0.251 & -0.017 \\
\hline Q4: E & Easily tired & 0.309 & 0.084 & -0.110 & 0.580 & 0.456 & 0.016 \\
\hline Q7: M & Headaches & 0.001 & 0.016 & -0.031 & 0.093 & 0.695 & 0.112 \\
\hline Q21:M & Menstrual cramps & 0.132 & 0.054 & -0.115 & 0.100 & 0.612 & 0.148 \\
\hline Q19: M & Abdominal bloating & 0.226 & 0.265 & 0.233 & 0.073 & 0.551 & 0.093 \\
\hline Q14:E & Felt frightened of getting cancer & -0.049 & 0.047 & 0.338 & 0.019 & 0.514 & 0.092 \\
\hline Q18: E & Self-conscious as result of PCOS & 0.039 & 0.221 & 0.045 & 0.249 & -0.032 & 0.671 \\
\hline Q20: M & Late menstrual period & 0.266 & 0.095 & -0.126 & -0.049 & 0.267 & 0.664 \\
\hline Q8: M & Irregular menstrual problems & 0.113 & -0.024 & -0.078 & -0.005 & 0.456 & 0.598 \\
\hline Q17: E & Worried about having PCOS & 0.074 & 0.093 & 0.424 & 0.364 & 0.274 & 0.544 \\
\hline Q23: I & Feel lack of control over the situation with PCOS & 0.102 & 0.122 & -0.006 & 0.511 & 0.271 & 0.521 \\
\hline
\end{tabular}

The factor loadings of the items all exceed 0.514 (given in bold font)

$H$ hirsutism, $W$ weight, I infertility, $E$ emotional and feelings, $M$ menstrual

Iran found similar results in achieving the reliability and validity in all domains with low value of correlation coefficient in the menstrual domains $[8,16,18]$. These low levels of achievement in the reliability and validity of the menstrual problem domain have been clarified differently. Guyatt et al. in Canada believes that the question regarding to headaches in the menstruation domain seemed not appropriate [18]. Bazarganipour et al. in Iran stated that relocating the question pointing to an irregular menstrual period (Question 8) from the emotional domain to the menstrual domain will significantly improved the reliability of both domains [17]. The menstrual domain in AR-PCOSQ will be assessed with more studies and utilization.

Test-retest reliability of the PCOSQ presented intraclass correlations above 0.9 in all the domains with $p$ value $<0.001$ (Table 3 ). That proved the stability and consistency of the PCOSQ over time. The period of two days to a week between the interviews was adopted to reduce recall bias while remaining within two weeks as stated by the questionnaires. Despite the fact that the second interview was done in another environment (at home through phone calls) while the initial assessment was in the obstetrics and gynecology clinics at KKUH, both were done by interviewing the participants to minimize the recall bias. Similar results of satisfactory intra-class correlation (ICC ranges from 0.71 to $0.92 ; p$ value $>0.05$ ) were reported in the Iranian version of PCOSQ [27].

Different items of PCOSQ loaded in different factors were noticed when the original English version of PCOSQ was translated to other languages or performed in various societies $[8,12,27]$. Our factor analysis of the AR-PCOSQ revealed six factors: emotions and feelings, body hair, weight, infertility, menstrual problems, and new factor. Two factors in our study (weight and body hair) were similar to the original scale of PCOSQ, with the same items loading on each factor. The infertility factor was identical except for one item ("feel a lack of 
control over the situation with PCOS") that loaded on a new factor. One item ("felt frightened of getting cancer) and other two items ("self-conscious as result of PCOS", and "Worried about having PCOS") of emotions and feeling factor were loaded on menstrual problems and the new factor, respectively. Two items ("late menstrual period" and "irregular menstrual problems") of menstrual problems factor were loaded into the new factor. These results are very similar to a study conducted by Jones et al. in the United Kingdom [8]. Therefore, moving some items from one factor to another may be considered. Bazarganipour et al. in the Iranian PCOSQ version moved the item "irregular menstrual problems" from the emotional factor to the menstrual factor, and the reliability of both factors improved significantly [27]. Therefore, we assume that classifying items such as "headaches", "menstrual cramps", "abdominal bloating", and "felt frightened of getting cancer" under the psychosomatic characteristics factor, and moving the other unmatched item to menstrual problems may improve the reliability of our study; especially the reliability of the menstrual problems factor.

Although the adequate sample size was sufficient to enable factor analysis and test-retest reliability, several limitations of our study need to be mentioned. First, all the participants were from one medical center and one nationality (Saudi Arabia). Most of them had a high educational level, [64.96\%, (Table 1)] and might have had good health literacy regarding their self-care and healthseeking behaviors. It is crucial for future researchers to validate the AR-PCOSQ using a sample from different regions and with subjects of various educational levels. Second, the responsiveness of the AR-PCOSQ was not assessed in this study due to the limited study period. Future research, along with sufficient follow-up time, should evaluate the ability of the AR-PCOSQ to describe the patient's health status over time and its sensitivity to detect changes in HRQoL because of the intervention, and to determine the minimal clinical importance score to justify whether changes are clinically relevant.

\section{Conclusions}

Our findings show the objective of establishing the initial reliability and validity of the Arabic version of the PCOSQ as a measure of the specific HRQoL in Saudi patients with PCOS. This will fill an important gap in measuring the HRQoL for patients with PCOS in research and community settings in Saudi Arabia. The PCOSQ is an excellent screening tool which health-care providers can be used to help prioritize health-related concerns from the patients' perspective.

\section{Abbreviations}

AR-PCOSQ: Arabic version of the PCOSQ; BMI: Body mass index; CVI: Content validity index; EFA: Exploratory factor analysis; HRQoL: Health-related quality of life; ICC: Intraclass correlation-coefficient; IRB: Institutional review board; KKUH: King Khalid University Hospital; KOM: Kaiser-Mayer-Oklin; PCOS: Polycystic ovarian syndrome; PCOSQ: Polycystic ovarian syndrome questionnaire.

\section{Acknowledgements}

The authors acknowledge the financial support from the Research Supporting Project (No. RSP-2020/16), King Saud University, Riyadh, Saudi Arabia.

\section{Authors' contributions}

SA, MA and YA designed the study, and analyzed and interpreted the patient data. FA \& AA collected the patients' data and performed the interview. SA was a major contributor in writing the manuscript. All authors read and approved the final manuscript.

Funding

Not applicable.

\section{Availability of data and materials}

The original (English) and translated (Arabic) polycystic ovarian syndrome questionnaire that support the findings of this study are available from McMaster University but restrictions apply to the availability of these questionnaires, which were used under license for the current study, and so are not publicly available. However, all data generated or analysed during this study are included in this published article.

\section{Ethical approval and consent to participate}

Ethical approval was granted by the institutional review board (IRB) at King Khaled University Hospital (IRB Project No. E-17-2545). The researcher explained the study verbally to all participants, and participants who agreed to participate gave verbal consent prior to interview. The verbal consent procedure was approved by the IRB.

\section{Consent for publication}

Participants were informed during the interview that the findings from their anonymised data may be used to write publications.

\section{Competing interests}

The authors declare that they have no competing interests.

\section{Author details}

${ }^{1}$ Basic Sciences Department, Prince Sultan College for Emergency Medical Services, King Saud University, Riyadh, Saudi Arabia. ${ }^{2}$ Department of Clinical Pharmacy, College of Pharmacy, King Saud University, P. O. Box 2457, Riyadh 11451, Saudi Arabia. ${ }^{3}$ Obstetrics and Gynecology Consultant, King Saud University Medical City, Riyadh, Saudi Arabia. ${ }^{4}$ Department of Obstetrics and Gynecology, College of Medicine, King Saud University, P. O. Box 2925, Riyadh 11461, Saudi Arabia.

Received: 1 October 2018 Accepted: 22 October 2020

Published online: 29 October 2020

\section{References}

1. Thathapudi S, Kodati V, Erukkambattu J, Katragadda A, Addepally U, Hasan Q. Anthropometric and biochemical characteristics of polycystic ovarian syndrome in South Indian women using AES-2006 criteria. Int J Endocrinol Metab. 2014;12(1):e12470.

2. Al-Ruhaily AD, Malabu UH, Sulimani RA. Hirsutism in Saudi females of reproductive age: a hospital-based study. Ann Saudi Med. 2008;28(1):28-32.

3. Ehrmann DA. Polycystic ovary syndrome. N Engl J Med. 2005:352:1223-36.

4. Hahn S, Janssen OE, Tan S, Pleger K, Mann K, Chedlowski M, et al. Clinical and psychological correlates of quality-of-life in polycystic ovary syndrome. Eur J Endocrinol. 2005;153:853-60. 
5. Kitzinger C, Willmott J. The thief of womanhood': women's experience of polycystic ovarian syndrome. Soc Sci Med. 2002;54:349-61.

6. Rasgon NL, Rao RC, Hwang S, Altshuler LL, Elman S, Zuckerbrow-Miller J, et al. Depression in women with polycystic ovary syndrome: clinical and biochemical correlates. J Affect Disord. 2003;74:299-304.

7. Legro RS, Arslanian SA, Ehrmann DA, Hoeger KM, Murad MH, Pasquali $R$, Welt CK. Diagnosis and treatment of polycystic ovary syndrome: an endocrine society clinical practice guideline. J Clin Endocrinol Metab. 2013:98(12):4565-92.

8. Jones GL, Hall JM, Balen AH, Ledger WL. Health-related quality of life measurement in women with polycystic ovary syndrome: a systematic review. Hum Reprod Update. 2008;14(1):15-25.

9. Naughton M, Shumaker S, Anderson R, Czajkowski S. Psychological aspects of health-related quality of life measurement: tests and scales. Quality of Life and Pharmacoeconomics in Clinical Trials. 2nd ed. Philadelphia: Lippincott-Raven 1996; 15:117-131.

10. Ou HT, Wu MH, Lin CY, Chen PC. Development of Chinese version of Polycystic Ovary Syndrome Health-Related Quality of Life Questionnaire (Chi-PCOSQ). PLOS ONE. 2015;10(10):e0137772.

11. Lin CY, Ou HT, Wu MH, Chen PC. Validation of Chinese version of Polycystic Ovary Syndrome Health-Related Quality of Life Questionnaire (Chi-PCOSQ). PLoS ONE. 2016;11(4):e0154343.

12. Oh J, Kim JH. Validity and reliability of a Korean version of Polycystic Ovary Syndrome Questionnaire. Korean J Women Health Nurs. 2014;20(4):255-65.

13. Nkosinathi N. Validation of the polycystic ovary syndrome health-related quality of life questionnaire (PCOSQ) in the clinical community in our gynaecological endocrine clinic; 2015. Retrieved January 26, 2017, from https://goo.gl/Vnfcug.

14. Jedel E, Kowalski J, Stener-Victorin E. Assessment of health-related quality of life: Swedish version of polycystic ovary syndrome questionnaire. Acta Obstet Gynecol Scand. 2008;87(12):1329-35.

15. Jones GL, Benes K, Clark TL, Denham R, Holder MG, Haynes TJ, et al. The Polycystic Ovary Syndrome Health-Related Quality of Life Questionnaire (PCOSQ): a validation. Hum Reprod. 2004;19(2):371-7.

16. Amini L, Ghorbani B, Montazeri A. Iranian version of Health-related Quality of Life for women with Polycystic Ovary Syndrome (PCOSQ): translation, reliability and validity [Abstract]. Iran Inst Health Sci Res. 2011;11(2):753-60.

17. Bazarganipour F, Ziaei S, Montazeri A, Foroozanfard F, Faghihzadeh S. Iranian version of modified polycystic ovary syndrome health-related quality of Life questionnaire: discriminant and convergent validity. Iran J Reprod. 2013;11(9):753-60.

18. Cronin L, Guyatt G, Griffith L, Wong E, Azziz R, Futterweit W, et al. Development of a health-related quality-of-life questionnaire (PCOSQ) for women with polycystic ovary syndrome (PCOS). J Clin Endocrinol Metab. 1998:83:1976-87.

19. Polit DF, Beck CT. Nursing research: principles and methods. 46th ed. Philadelphia: Lippincott; 2004. p. 416-45.

20. Jenkinson C, Mcgee H. Health status measurement: a brief but critical introduction. Oxford: Radcliffe Medical Press; 1998.

21. Cronbach L. Coefficient alpha and the internal structure of tests. Psychometrika. 1951;16:297-334.

22. Landis JR, Koch GG. The measurement of observer agreement for categorical data. Biometrics. 1977;33:159-74.

23. Kline P. A psychometrics primer. London: Free Association Books; 2000.

24. Cerny BA, Kaiser HF. A study of a measure of sampling adequacy for factor-analytic correlation matrices. Multivar Behav Res. 1977;12(1):43-7.

25. Kaiser H. An index of factor simplicity. Psychometrika. 1974;39:31-6.

26. Gliem JA, Gliem RR. Calculating, interpreting, and reporting Cronbach's alpha reliability coefficient for Likert-type scales. In: 2003 Midwest research-to-practice conference in adult, continuing, and community education. 2003:82-8.

27. Bazarganipour F, Ziaei S, Montazeri A, Foroozanfard F, Faghihzadeh S. Psychometric properties of the Iranian version of modified polycystic ovary syndrome health-related quality-of-life questionnaire. Hum Reprod. 2012;27(9):2729-36

\section{Publisher's Note}

Springer Nature remains neutral with regard to jurisdictional claims in published maps and institutional affiliations.
Ready to submit your research? Choose BMC and benefit from:

- fast, convenient online submission

- thorough peer review by experienced researchers in your field

- rapid publication on acceptance

- support for research data, including large and complex data types

- gold Open Access which fosters wider collaboration and increased citations

- maximum visibility for your research: over $100 \mathrm{M}$ website views per year

At $\mathrm{BMC}$, research is always in progress.

Learn more biomedcentral.com/submissions 